\title{
Lixiviats Issus De La Décharge Publique De La Ville De Meknès: Calcul Et Résultat Du Bilan Hydrique
}

\author{
Lekehal Hicham, Dr. \\ Bouchelta Aziz, PhD
}

Université Moulay Ismail, Faculté des Sciences, département de Biologie, Laboratoire de recherche Environnement \& Santé Dép. Biologie B.P 11201 Zitoune Meknès, Maroc.

Benzougagh Brahim, Dr.

Université Moulay Ismail, Faculté des sciences Département de Géologie, Laboratoire de Géoscience, Patrimoine et substances utiles (GPS). B.P 11201

Zitoune, Meknès- Maroc

\section{Mimich Khalid, Dr.}

Université Moulay Ismail, Faculté des sciences Département de Géologie, Equipe Sciences de l'Eau et Ingénierie Environnementale. B.P 11201

Zitoune, Meknès- Maroc

doi: 10.19044/esj.2016.v12n29p242 URL:http://dx.doi.org/10.19044/esj.2016.v12n29p242

\begin{abstract}
The leachate issued from the garbage dumps of the city of Meknes represents a great environmental threat as they are neither recovered nor treated before their discharge into the natural environment, thus constituting an important source of pollution to the water of the Boufekrane's river and neighboring agricultural land to the dump. Thus is the need to quantify the rate of this type of effluent: in order to offer technical and economic solutions to its treatment or disposal. The amount of waste arriving at the dump is estimated to 554 tons per day in 2015 with $40 \%$ average moisture content and a loss of water in the percolate from 5 to $10 \%$, thus the amount of water supplied by the waste is estimated at $88.8 \mathrm{~m} 3$ per day. The volume of runoff water from the inside of the dump to the outside is estimated to be $38 \mathrm{~m} 3$ per day while the volume from outside to inside is null or negligible given the topography. The infiltration in soil is negligible because the floor of the dump consists of lithological marl Miocene formations, rendering the dump waterproof. The area, consisting of 17 hectares, receives an effective average rainfall of $475 \mathrm{~mm}$ / year, producing a volume of $80750 \mathrm{~m} 3$ / year, equivalent to $221 \mathrm{~m} 3$ / day. The total volume of leachate is about $271 \mathrm{~m} 3$ per day.
\end{abstract}


Keywords: Leachates, dumps, water, pollution, infiltration, Meknes.

\section{Résumé}

Les lixiviats issus de la décharge publique de la ville de Meknès Présentent une grande menace environnementale du fait qu'ils ne sont ni récupérés ni traités avant leur rejet en milieu naturel, constituant ainsi, une source de pollution importante des eaux de l'oued Boufekrane et des terrains agricoles limitrophes à la décharge. D'où la nécessité de quantifier le débit de ce type d'effluent afin de proposer les solutions technico-économiques de son traitement ou élimination.

La quantité des déchets arrivant à la décharge est estimée à 554 tonnes par jour en 2015 avec un taux moyen d'humidité de $40 \%$ et une perte d'eau dans le percolât de 5 à 10\%, ainsi, la quantité d'eau apportée par les déchets est de l'ordre de $88,8 \mathrm{~m}^{3} / \mathrm{j}$. Le volume d'eau de ruissellement de l'intérieur de la décharge vers l'extérieur est estimée à $38 \mathrm{~m}^{3} / \mathrm{j}$ tandis que celui de l'extérieur du site vers l'intérieur est nul ou négligeable vu la topographie du terrain. L'infiltration dans le sol est négligeable car le sol de la décharge est constitué par des formations lithologiques marneuses du Miocène, ce qui rend la décharge parfaitement étanche. Le site, d'une superficie de 17 hectares, reçoit une pluie efficace moyenne de $475 \mathrm{~mm} / \mathrm{an}$, produisant ainsi un volume de $80750 \mathrm{~m}^{3} / \mathrm{an}$, équivalent à $221 \mathrm{~m}^{3} / \mathrm{j}$. Le volume total de lixiviats produits est donc d'environ $271 \mathrm{~m}^{3} / \mathrm{j}$.

Mots Clés : Lixiviats, décharge publique, eau, pollution, infiltration, Meknès

\section{Introduction}

Les déchets, produits par les ménages et par les activités économiques, sont générateurs de nuisances et peuvent être dangereux pour l'homme et l'environnement. Il est donc essentiel d'en contenir la production et d'en maîtriser le devenir.

Les lixiviats principaux sources de polluants, sont issus de la percolation de l'eau à travers le massif des déchets. Leur composition varie avec les facteurs suivants :

- La composition des déchets ;

- L'âge du site et des déchets, soit l'état d'avancement de la dégradation ;

- Le bilan hydrique qui détermine les quantités d'eau reçues et de lixiviats produits ;

- Le mode d'exploitation qui régi les conditions régnant au sein du massif des déchets. 
Les eaux de pluie percolant à travers la décharge de Meknès ne sont ni collectées ni drainées par un réseau spécifique. Leur volume dépend de l'infiltration efficace des précipitations.

Le volume de lixiviats est estimé par le calcul du bilan hydrique qui prend en compte :

- $\quad$ Les précipitations qui constituent l'apport brut d'eau à la surface de la décharge ;

- $\quad$ Le ruissellement qui désigne le phénomène d'écoulement des eaux à la surface des sols;

- L'évapotranspiration réelle, responsable du prélèvement d'une partie du volume apporté par les précipitations.

Dans le cadre de cette problématique environnementale, l'objectif de cette recherche est de quantifier le volume des lixiviats produits par la décharge de Meknès pour proposer les solutions technico-économique de leurs traitements.

\section{Présentation de la zone d'étude}

\section{Contexte géologique}

Il occupe la partie sud-ouest du bassin de Saïss (fig.01). Il a une forme semi-triangulaire avec une surface deux fois plus grande que son homologue nord-oriental. Sa plus grande dimension est d'environ $60 \mathrm{~km}$ d'ouest en est, s'accompagne également d'un relèvement assez remarquable matérialisé par un doux étagement du plateau depuis les $500 \mathrm{~m}$ de la localité de Aïn Orma à l'ouest, jusqu'aux 700m (fig.02) à la pointe du Bitit (Fassi, 1999). La différenciation de la pente entre le Nord et le Sud, influence la morphologie des cours d'eau et la vitesse de circulation des eaux souterraines (Amraoui, 2005).

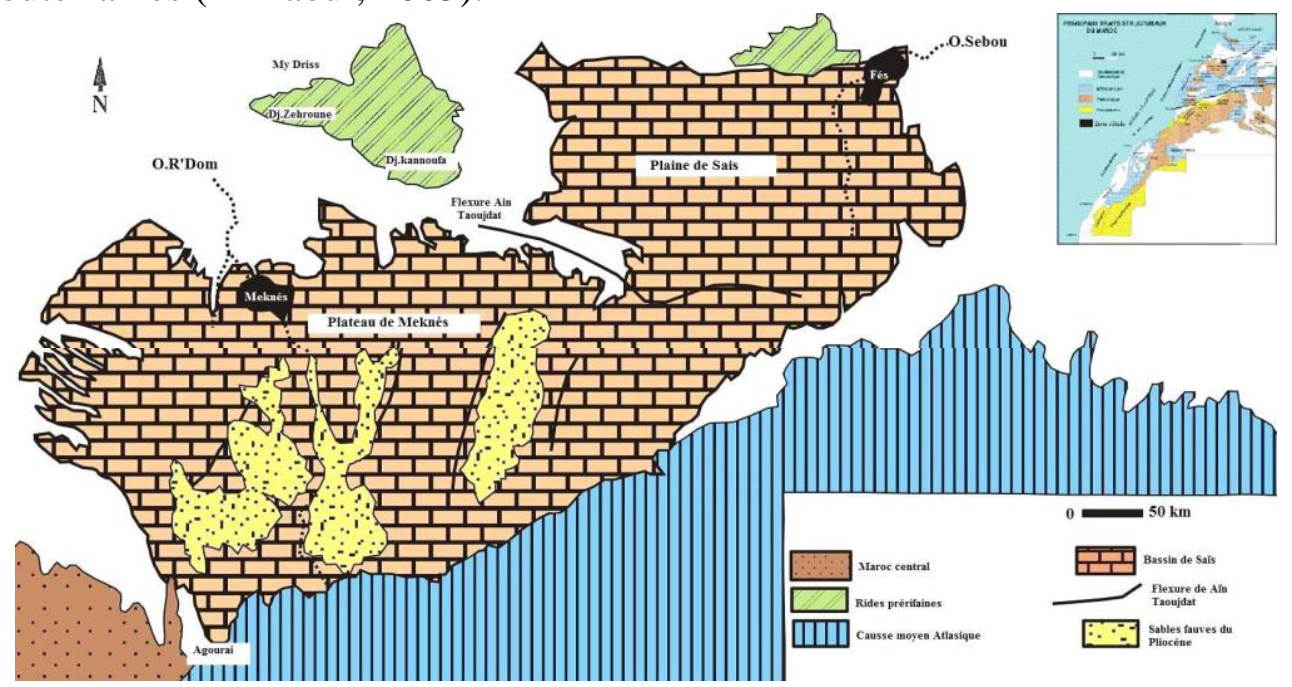

Figure1 : Esquisse géologique du bassin de Saïss (Taltasse, 1953). 


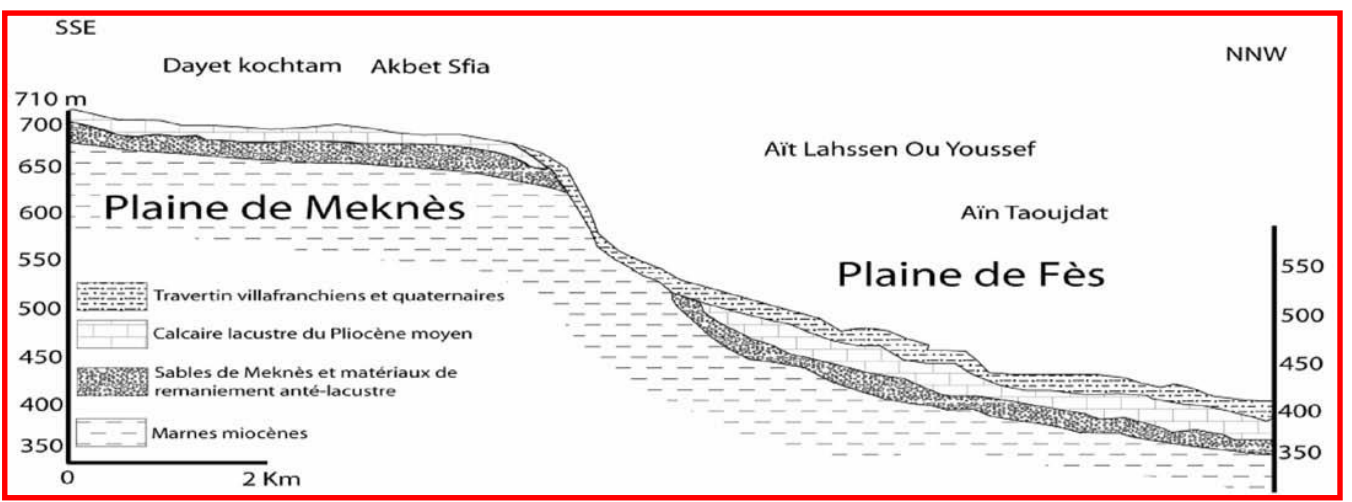

Figure 2 : Différenciation d'altitude entre la plaine de Fès et plateau de Meknès (Fassi,

\section{Lithostratigraphie} 1999).

La zone d'étude se situe dans le bassin de Saïss et particulièrement dans le plateau de Meknès, aux coordonnées Lambert X : 483600 et Y : 370000, (fig.03).

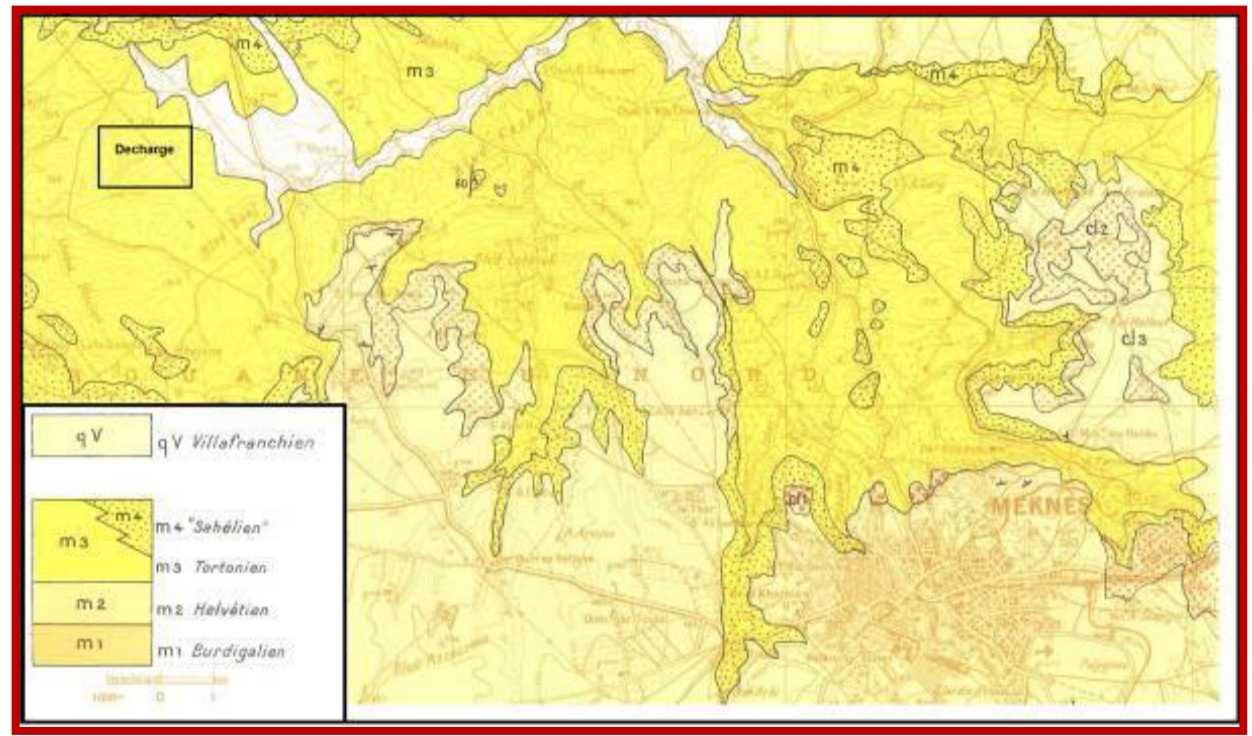

Figure 3 : Situation de la décharge sur la carte géologique de Meknès au /100 000.

Le bassin de Saïss est une large structure sédimentaire tertiaire à remplissage miocène et plio-quaternaire, mais la lithostratigraphie débute dès le Paléozoïque (fig.04). Les variations à noter sont celles des formations Plio-quaternaires dans le bassin qui varient selon le contexte sédimentaire local (Harmouzi, 2010). 


\section{Paléozoïque}

Le Paléozoïque est représenté par une alternance de pélites et de bancs gréseux. Il affleure au massif hercynien central où il est en contact large avec le bassin au Sud. Le socle paléozoïque affleure au sud-ouest de la ville de Meknès au niveau d'Oued El Kell à la région d'Agouray, au niveau d'El Bhalil et au niveau d'El Hajeb.

\section{Trias}

Il est constitué essentiellement par des argiles gypsifères et salifères (dépôts évaporitiques) a intercalations de basaltes dolomitiques (fig.04). Un niveau sédimentaire carbonaté, de faible épaisseur ( $5 \mathrm{~m}$ au maximum), est en permanence associé à des basaltes (Ourghache, 1987).

\section{Lias}

Le Lias du Saïss n'est connu qu'à travers les sondages réalisés dans le bassin, à l'exception de sa bordure sud-est où il arrive à l'affleurement à Bou Oughioul et montre des faciès proches de ceux des Causses (Amraoui, 2005). Les formations liasiques constituent le matériel essentiel des reliefs avec des calcaires dolomitiques inférieurs massifs et ruiniformes du Lias inférieur (Lotharingien), surmontées par des calcaires à céphalopodes et calcaires à silex du Lias moyen constitué par les étages Carixien et Domérien. Elles présentent une puissance très variable (Essahlaoui, 2000). L'épaisseur de ces formations retrouvées dans certains forages peut atteindre 280m (région de Sebâa Ayoun). Les profondeurs de ces formations sont de plus en plus grandes en allant du sud vers le nord.

\section{Miocène supérieur}

La colonne stratigraphique (fig.04), montre que la série miocène est généralement marneuse. Le passage du Lias au Miocène est matérialisé par un conglomérat de base, puis un calcaire gréseux a passées marneuses, et le reste dominé par les marnes grises du Tortonien. La limite entre le Tortonien et le Missinien est marquée par une strate de grès, surmontée par les marnes grises gréseuses (Aït Brahim, 1991 \& Essahlaoui, 2000).

\section{Pliocène}

Le Pliocène inférieur est représenté par un dépôt de sables à matrice carbonatée avec une épaisseur de 50-80m dans la région de Meknès (Boumir, 1987). Il est surmonté par des sables fauves du Pliocène moyen, d'une trentaine de mètre, déposé sur le plateau de Meknès (fig.04). Ces sables sont de couleur variable (rouge, jaune, brun, ocre, gris...etc) et constituent les futurs sables fauves (Taltasse, 1953). Le Pliocène supérieur, représenté par 
une alternance des calcaires argileux et de calcaires micritiques, est connu sous le nom de calcaires lacustres (Taltasse, 1953).

\section{Quaternaire}

La colonne se termine par un Quaternaire très diversifié (fig.04), constitué d'un complexe fluviatile (Ahmamou, 1987). Ce complexe est constitué par des tufs, des conglomérats, des lentilles de calcaires et des limons gris.

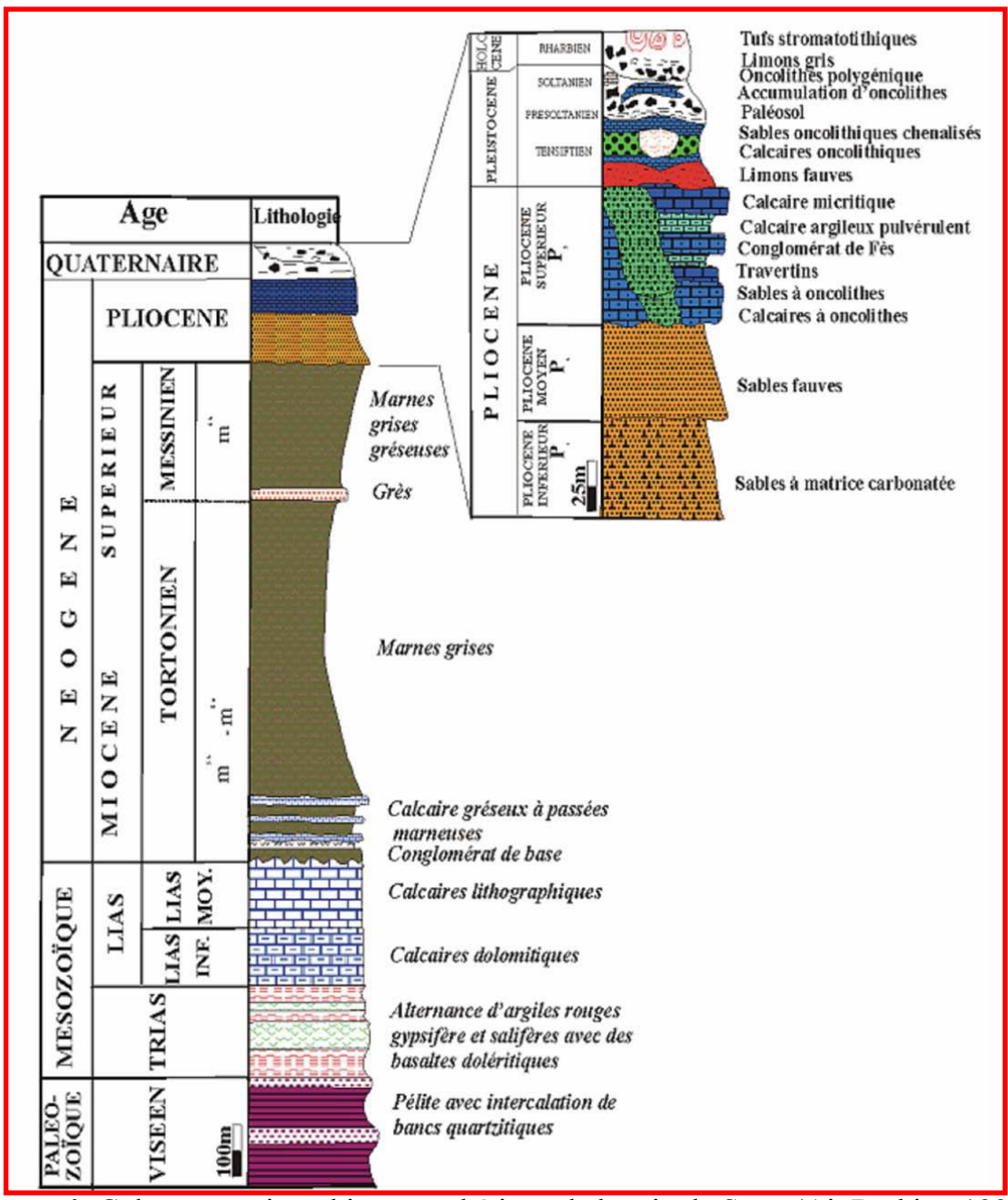

Figure 4: Colonne stratigraphique synthétique de bassin de Saïss (Ait Brahim, 1991).

Les forages réalisés dans le site lors de l'élaboration de l'étude d'impact sur l'environnement de la décharge publique de Meknès confirment les études géologiques de surface. En effet d'après la reconnaissance in situ réalisée au moyen de 3 sondages carottés, on trouve sous la terre végétale $(20 \mathrm{~cm})$ 
jusqu'à $20 \mathrm{~m}$ les marnes argileuses limoneuses compacte en profondeur. Ces marnes peuvent dépasser 2km d'épaisseur dans le bassin de Sais (Sita, 2013).

\section{Contexte hydrologique hydrogéologique}

Le réseau hydrographique au niveau du plateau de Meknès (fig.05), présente une direction qui coïncide avec la direction de la surface structurale sus-sus-est et nord-nord-ouest. La zone d'étude (décharge de Meknès) se situe dans le bassin versant du Rdom comprenant les oueds : Boufekrane, Ouisslane et Chajara. Le plus important de ces oueds est Boufekrane, aussi bien en raison de son débit ( 310 1/s en étiage) que de son déploiement au cœur de la ville (Abdouh et al, 2004). La majorité du site de décharge est drainée par l'Oued Boufekrane. On constate la présence, à l'intérieur de la décharge, de 2 thalwegs qui rejoignent l'Oued Boufekrane. Le thalweg Sud, le plus important, est partiellement rempli par les déchets. Du point de vue hydrogéologique, on distingue dans le bassin de Meknès -Fès deux nappes importantes. Il s'agit d'une part de la nappe phréatique d'age plioquaternaire et de la nappe profonde du Lias (Tabyaoui, 2005).Comme le montre la figure 05 , le site de la décharge est situé sur une zone imperméable de la nappe phréatique principale.

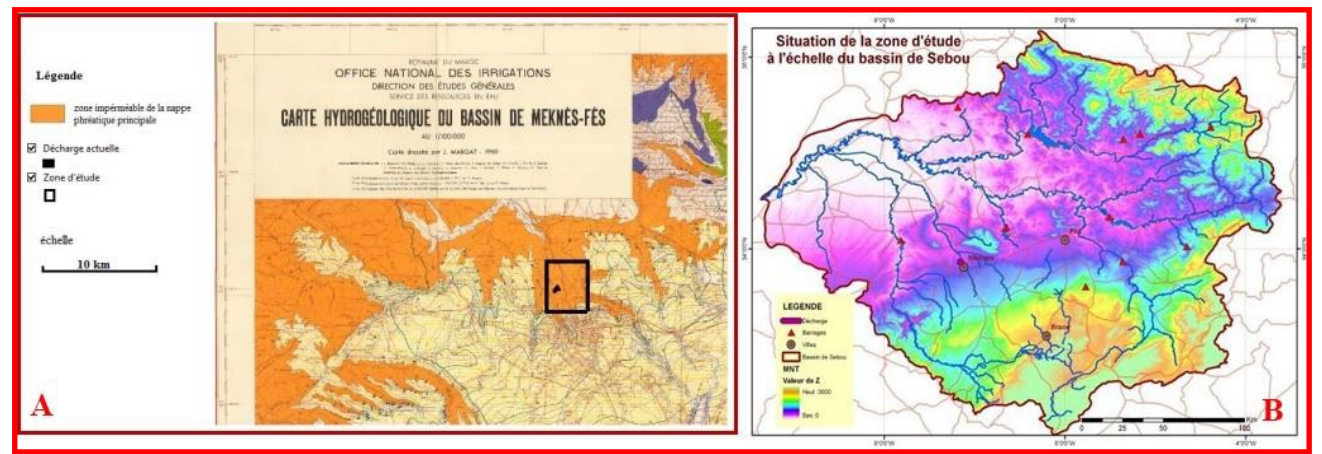

Figure 5 : le contexte et hydrogéologique (A) (Margat, 1960) et hydrologique (B) du bassin du Saïss.

\section{Description de la décharge}

La décharge de la ville de Meknès est une décharge non contrôlée desservant la totalité de la zone urbaine de Meknès. Elle a été mise en service en l'an 2002. La surface disponible pour les besoins de la mise en décharge est à l'ordre de 53 ha, dont 17 ha actuellement occupée par les déchets. La décharge se situe à environ $5 \mathrm{~km}$ à vol d'oiseau au nord-ouest de la ville, à moins de $8 \mathrm{~km}$ du centre-ville et à $1,5 \mathrm{~km}$ de la route nationale, aux coordonnées Lambert (x : 483600 et y : 370000), localisée au niveau de la zone rurale de Dkhissa, adjacente à la commune rurale de Dar Oum Sultane. L'accès à la décharge se fait via la route principale vers Sidi Kacem sur une 
distance de $6,5 \mathrm{Km}$ à partir de la ville de Meknès suivie d'une piste en toutvenant de 1,5 Km (SITA, 2013).

L'exploitation des données communiquées permet d'estimer le tonnage en 2015 produit par la ville de Meknès à 202116 tonnes/an, soit une production journalière moyenne de 554 tonnes /jour. En 2031 ce tonnage sera de l'ordre de 304027 tonnes/an, soit 8 une production journalière d'ordre de 833 tonnes/jour. La population en 2031 sera de 1'ordre de 895570 habitants, ainsi le tonnage cumulatif prévisionnel qui sera produit est estimé à 4844425 Tonnes (tab.01)

Tableau 1: Evolution de la production des déchets solides de Meknès.

\begin{tabular}{cccc}
\hline Année & Population $($ Hab. & Tonnage journalier $(\mathrm{T} / \mathrm{j})$ & Tonnage annuel (T/an) \\
\hline 2012 & 644695 & 509 & 185624 \\
2013 & 658902 & 525 & 191462 \\
2014 & 670789 & 539 & 196720 \\
2015 & 682924 & 554 & 202116 \\
2016 & 695313 & 569 & 207655 \\
2017 & 707964 & 584 & 213340 \\
2018 & 720882 & 600 & 219175 \\
2019 & 734074 & 617 & 225165 \\
2020 & 747547 & 634 & 231314 \\
2021 & 761309 & 651 & 237626 \\
2022 & 775366 & 669 & 244107 \\
2023 & 789726 & 687 & 250761 \\
2024 & 802138 & 704 & 256897 \\
2025 & 814774 & 721 & 263174 \\
2026 & 827639 & 739 & 269598 \\
2027 & 840738 & 757 & 276170 \\
2028 & 854075 & 775 & 282896 \\
2029 & 867656 & 794 & 289778 \\
2030 & 881485 & 813 & 296820 \\
2031 & 895570 & 833 & 304027 \\
TONNAGE TOTAL PREVISIONNEL SUR 20 ans & 4844425 \\
\hline
\end{tabular}

Pour la composition des déchets dans la ville de Meknès, la fraction biologique représente environ $70,7 \%$, celle relative au papiers/cartons représente environ $6 \%$, tandis que la fraction plastique représente environ 7,4\%. Les autres fractions représentent 15,9\%.(GTZ, 2010).

\section{Impact du site actuel sur l'environnement}

Dans la décharge actuelle, les déchets sont déversés à même le sol sans prendre aucune précaution particulière vis-à-vis de la protection de l'environnement ni de la stabilité générale du site et des dépôts. Les dépôts de déchets ont atteints une épaisseur très importante notamment à l'angle SUD-EST où la plate-forme de déchets culmine à l'altitude $440 \mathrm{~m}$, soit plus 
de 10 à 15 mètres au-dessus du terrain naturel formant un point haut et une ligne de partage des eaux (SITA, 2013).

Ainsi la morphologie du site qui initialement présentait une topographie en talweg avec un écoulement des eaux centralisé et dirigé en direction de l'Oued Boufekrane à l'Ouest a désormais beaucoup changé et une deuxième ligne d'écoulement des eaux a été créée en direction de l'oued Ouisslane à l'Est. L'impact sur les eaux superficielles est donc fort puisque les lixiviats qui ne peuvent pas s'infiltrer dans le sous-sol ruissellent selon ces deux lignes d'écoulement et impactent directement les deux oueds après avoir traversé des zones de cultures et de pâtures. Sur le flanc Ouest, la majeure partie des écoulements des lixiviats converge rapidement vers l'Oued Boufekrane (SITA, 2013).

Méthodologie de travail

\section{Estimation du débit des lixiviats à travers le bilan hydrique de la décharge}

La quantité des lixiviats produite a été déduite du bilan hydrique de la décharge. Ce bilan met en jeu les entrées et les sorties de l'eau dans cette décharge, comme le montre l'équation utilisée par Matejka et Rink, Leclerc et Bonneau et Navarro. Et comme l'indique le schéma suivant (fig.06) :

$\mathrm{P}+\mathrm{E}_{\mathrm{D}}+\mathrm{R}_{1}=\mathrm{I}+\mathrm{E}+\mathrm{R}_{2}+\mathrm{ETR}$

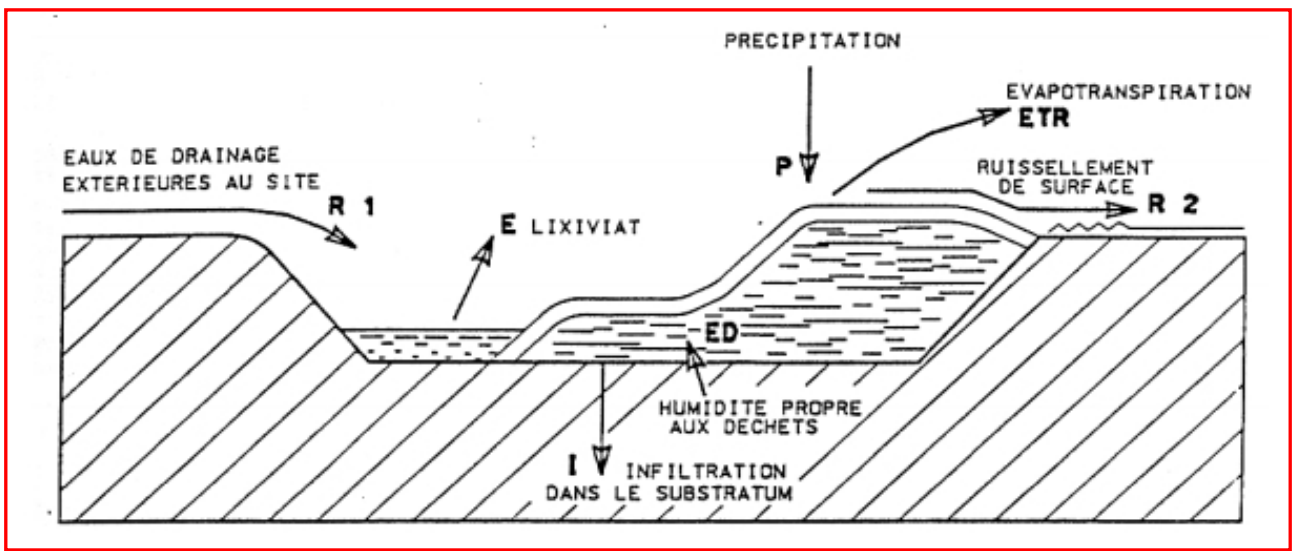

Figure 6 : Schéma du bilan hydrique de la décharge (http://www.diastratasa.com/environnement/decharges.htm).

Le volume de lixiviats susceptible d'être collecté au niveau de la décharge est donné par l'équation suivante :

$$
\mathbf{E}=(\mathbf{P}-\mathbf{E T R})+\mathbf{E}_{\mathbf{D}}+\mathbf{R}_{\mathbf{1}}-\mathbf{I}-\mathbf{R}_{2}
$$

Avec:

$$
\text { P : Précipitations ; }
$$


- ETR : Eau réelle transpirée et évaporée directement ou en raison d'élévation de la température du à l'évolution biologique ;

- P-ETR $\left(\mathrm{m}^{3} / \mathrm{an}\right) \quad$ : Pluie efficace.

- ED : Eau apportée par les déchets

- R1 : Eaux de ruissellement: eaux de surface de l'extérieur du site vers l'intérieur ;

- I $\quad$ : Eau d'infiltration dans le sol ;

- E : Lixiviats Produits ;

- $\mathbf{R}_{2} \quad$ : Eau de ruissellement de l'intérieur de la décharge vers l'extérieur, donnée par l'équation suivante :

$\mathbf{R}_{\mathbf{2}}=\mathbf{k} *(\mathbf{A} * \mathbf{P}) / \mathbf{1 0 0 0}$

Avec :

- A : aire arrosée ;

- P:pluviométrie ;

- $\quad \mathbf{K}$ : coefficient de percolation des eaux pluviales

Le volume final des lixiviats susceptible d'être collecté est :

$\mathbf{E}=(\mathbf{P}-\mathbf{E T P})+\mathbf{E}_{D}-\mathbf{R}_{2}$

\section{Résultats et discussion}

\section{Bilan hydrique}

\section{Précipitations}

La pluviométrie moyenne (calculée sur la période 1934 - 2001) dans la ville de Meknès atteint $559 \mathrm{~mm} /$ an avec un écart type de $145 \mathrm{~mm}$ et une variation de $25.9 \%$.(fig.07).

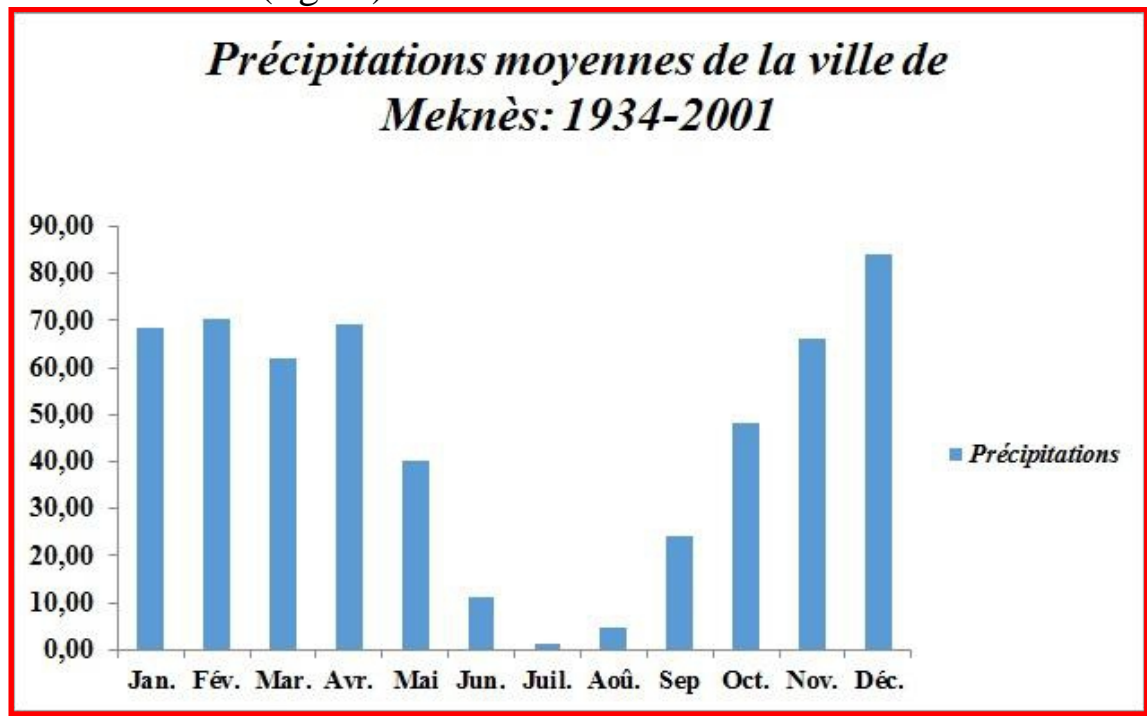

Figure 7: Pluies mensuelles moyennes (1934-2001) (CID/SEURECA, 2006). 
Eau réelle transpirée et évaporée

En l'absence d'une méthode de calcul ETR spécifique aux décharges, les déchets ont été assimilés à un sol. ETR a été inclus dans le calcul de la pluie efficace.

\section{Pluie efficace}

La pluie efficace $(\mathbf{P}-\mathbf{E T R})$ est égale à la différence entre les précipitations totales et l'évapotranspiration réelle. Elle est calculée directement à partir des paramètres climatiques et de la réserve utile du sol $(\mathrm{RU}) . \mathrm{L}$ 'eau de cette pluie est répartie au niveau du sol, entre l'écoulement superficiel et l'infiltration. Elle a été calculée par l'application CROPWAT de la FAO (fig.08).

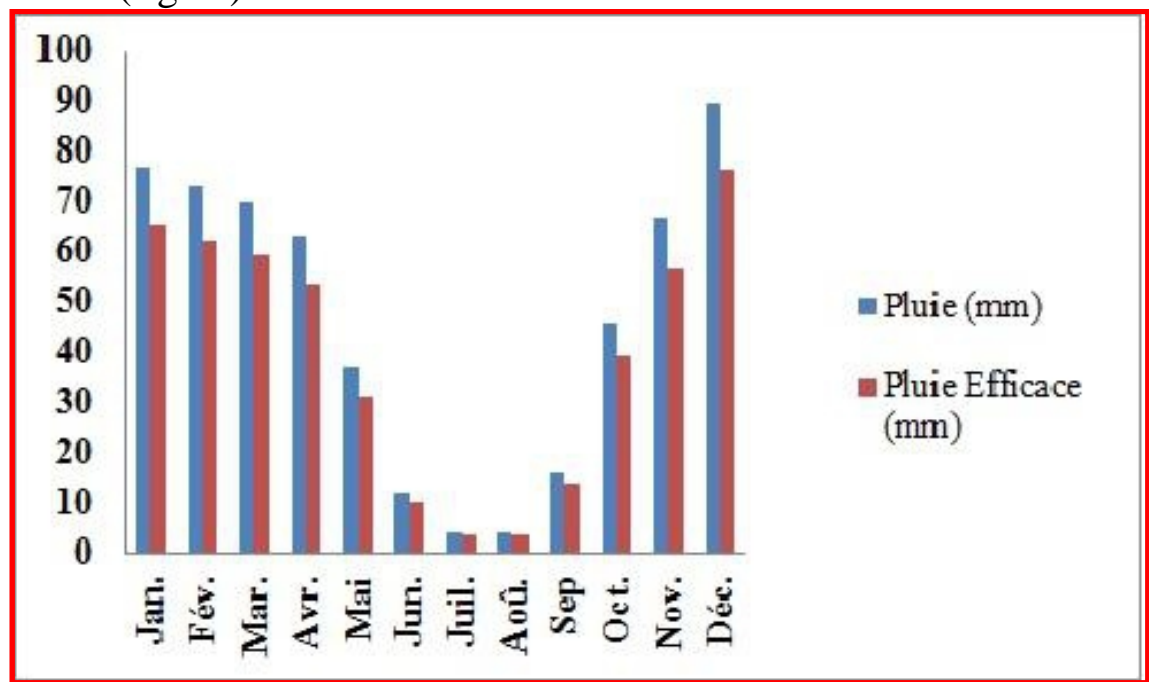

Figure 8 : Pluie efficace (P-ETR)

\section{Eau apportée par les déchets}

Le calcul du volume d'eau apporté par les déchets $\mathbf{E}_{\mathbf{D}}$ prend en considération l'évolution de la production des déchets solides de la ville de Meknès due essentiellement à l'accroissement démographique. Pour cela un tonnage prévisionnel a été établi sur une période de 20 ans. Il y’a lieu de prendre en considération le taux moyen d'humidité qui est de $40 \%$ avec une perte dans le percolât de 5 à $10 \%$ de la quantité d'eau contenue dans les déchets et ce dû à leur compactage (fig.09). 


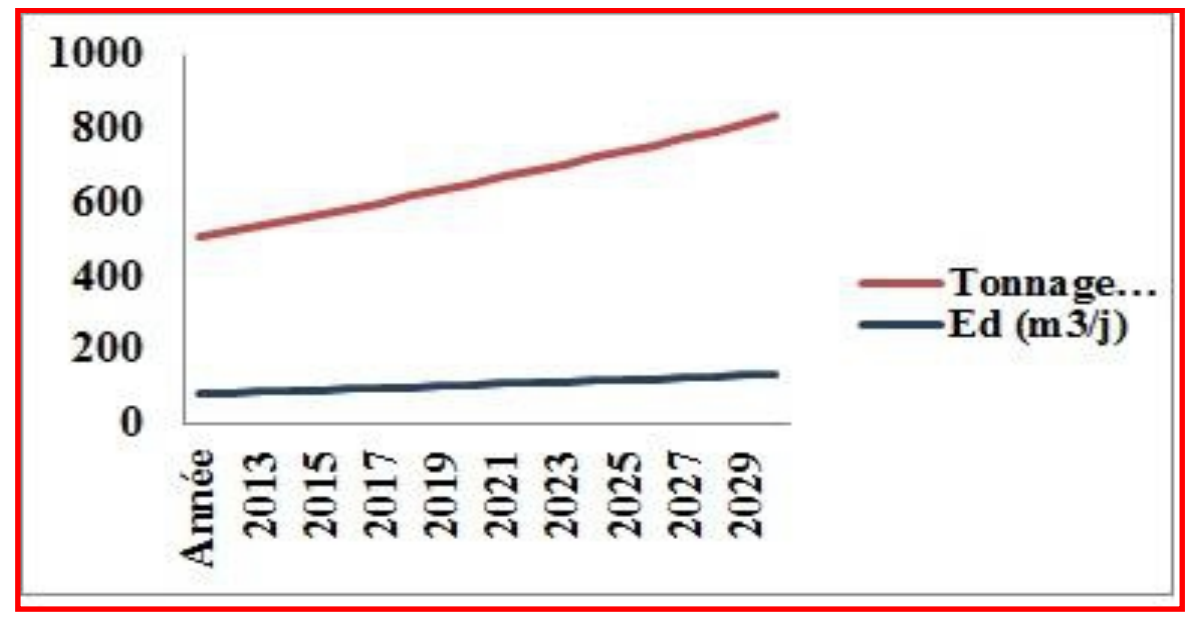

Figure 9 : Evolution du volume d'eau Eau apportée par les déchets $\left(\mathrm{E}_{\mathrm{D}}\right)$ sur une période de 20 ans.

Eaux de ruissellement de l'extérieur du site vers l'intérieur

Dans le cas de la décharge de Meknès, R1 est nul ou négligeable vu la topographie du terrain (terrain accidenté).

\section{Eau d'infiltration dans le sol}

I est négligeable car le sol de la décharge est constitué par des formations lithologiques marneuses du Miocène, ce qui rend la décharge parfaitement étanche.

\section{Eau de ruissellement de l'intérieur de la décharge vers l'extérieur}

\section{$\mathbf{k} *(\mathbf{A} * \mathbf{P}) / \mathbf{1 0 0 0}$}

Le tableau 02 récapitule la valeur de $\mathbf{R 2}$ donnée par l'équation : $\mathbf{R 2}=$ Avec :

A : aire arrosée de 17 hectares;

$\mathbf{P}$ : pluviométrie qui varie selon les périodes de l'année ;

$\mathbf{K}$ : coefficient de percolation des eaux pluviales de l'ordre de 0,15 . Le coefficient de ruissellement qui est le rapport du volume d'eau ruisselée par le volume d'eau tombée, est généralement assimilé au taux d'imperméabilisation du site qui est égal au rapport de la surface imperméabilisée par la surface totale. Le coefficient de ruissellement Adopté est de l'ordre de 0,85

Tableau 2 : Volume d'eau de ruissellement de l'intérieur de la décharge vers l'extérieur

\begin{tabular}{cccccccccccccc}
\hline & Jan & Fév. & $\begin{array}{c}\text { Mar } \\
\text {. }\end{array}$ & $\begin{array}{c}\text { Mv } \\
\text { r. }\end{array}$ & $\begin{array}{c}\text { Ma } \\
\text { i }\end{array}$ & $\begin{array}{c}\text { Jun } \\
\text { Juil }\end{array}$ & $\begin{array}{c}\text { Ao } \\
\text { u. }\end{array}$ & $\begin{array}{c}\text { Se } \\
\text { p. }\end{array}$ & Oct. & Nov & $\begin{array}{c}\text { Dé } \\
\text { c. }\end{array}$ & $\begin{array}{c}\text { Tota } \\
1\end{array}$ \\
\hline R2 & 4,7 & 4,90 & 4,32 & 4,8 & 2,8 & 0,7 & 0,0 & 0,3 & 1,7 & 3,37 & 4,61 & 5,8 & 38,4 \\
m3 & 7 & 8 & 3 & 2 & 2 & 9 & 8 & 4 & & 2 & 7 & 7 & 1 \\
/j & & & & & & & & & & & & & \\
\hline
\end{tabular}




\section{Volume des Lixiviats produits}

Le flux des lixiviats calculé théoriquement par le biais du bilan hydrique (tab.03) évolue avec le temps suite à l'augmentation du volume d'eau apporté par les déchets. Ce résultat devrait être vérifié en effectuant des mesures expérimentales sur le terrain, chose qui s'est avérée difficile du fait que l'écoulement des lixiviats est diffus. Plusieurs filons de débits insignifiants ont été identifiés, ils convergent vers des endroits différents. Le seul filon de lixiviat qui a pu être mesuré provient du bassin de rétention, son débit a été estimé à $0,21 / \mathrm{s}$ (débit estimé à travers la vitesse d'écoulement du filon, le niveau d'eau dans le filon et sa largeur).Ce débit est loin de représenter le débit réel généré par la décharge (tab.03-fig.10).

Tableau 3 : Evolution du volume des lixiviats produits

\begin{tabular}{lccccc}
\hline Année & Tonnage journalier(T/j) & P-ETP & $\mathrm{R} 2$ & $\mathrm{Ed}\left(\mathrm{m}^{3} / \mathrm{j}\right)$ & $\mathrm{E}\left(\mathrm{m}^{3} / \mathrm{j}\right)$ \\
\hline 2012 & 509 & 9,22 & 38,41 & 81,36 & 264,24 \\
2013 & 525 & 9,22 & 38,41 & 83,92 & 266,80 \\
2014 & 539 & 9,22 & 38,41 & 86,23 & 269,11 \\
2015 & 554 & 9,22 & 38,41 & 88,59 & 271,47 \\
2016 & 569 & 9,22 & 38,41 & 91,02 & 273,90 \\
2017 & 584 & 9,22 & 38,41 & 93,51 & 276,39 \\
2018 & 600 & 9,22 & 38,41 & 96,07 & 278,95 \\
2019 & 617 & 9,22 & 38,41 & 98,70 & 281,58 \\
2020 & 634 & 9,22 & 38,41 & 101,39 & 284,27 \\
2021 & 651 & 9,22 & 38,41 & 104,16 & 287,04 \\
2022 & 669 & 9,22 & 38,41 & 107 & 289,88 \\
2023 & 687 & 9,22 & 38,41 & 109,92 & 292,80 \\
\hline 2024 & 704 & 9,22 & 38,41 & 112,61 & 295,49 \\
2025 & 721 & 9,22 & 38,41 & 115,36 & 298,24 \\
2026 & 739 & 9,22 & 38,41 & 118,17 & 301,05 \\
2027 & 757 & 9,22 & 38,41 & 121,06 & 303,94 \\
2028 & 775 & 9,22 & 38,41 & 124 & 306,88 \\
2029 & 794 & 9,22 & 38,41 & 127,02 & 309,90 \\
2030 & 813 & 9,22 & 38,41 & 130,11 & 312,99 \\
2031 & 833 & 9,22 & 38,41 & 133,27 & 316,15 \\
\hline & & & & & \\
\hline
\end{tabular}




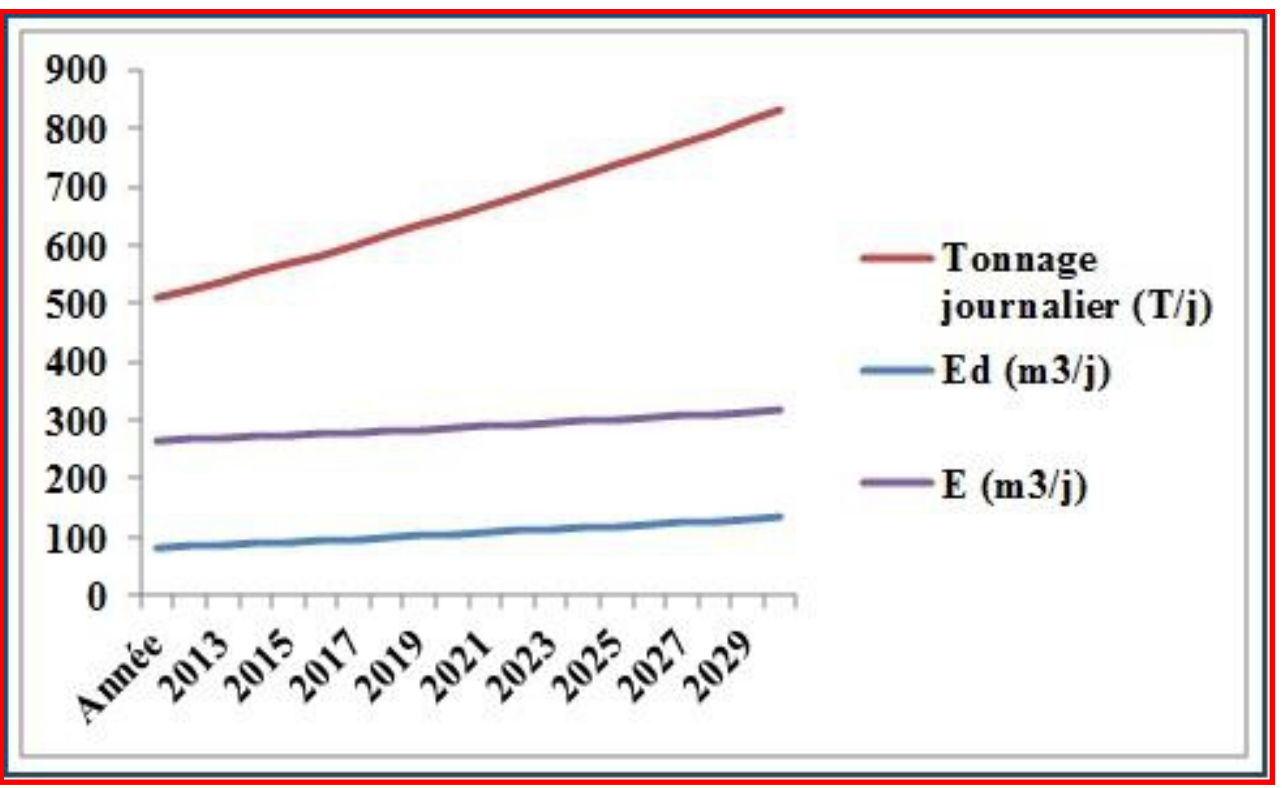

Figure 10 : Evolution du volume des lixiviats produit

\section{Conclusion}

Pour une gestion responsable de la décharge de la ville de Meknès, l'estimation du flux des lixiviats s'avère nécessaire. Le volume des lixiviats calculé par le biais du bilan hydrique évolue d'une façon remarquable et atteindra environ $317 \mathrm{~m}^{3} /$ jour en 2031 , un résultat dépendant de plusieurs facteurs tel que la pluviométrie et l'augmentation de la production des déchets par habitant et par jour. Reste à effectuer une caractérisation physico-chimique et bactériologique des lixiviats afin de proposer les solutions technico-économique pour remédier à ce problème.

\section{References:}

1. AHMAMOU, H., (1987). Etude sédimentologique des calcaires lacustres Saïssiens (Plio-Quaternaire) du bassin de Fès-Meknès (Maroc). Thèse de 3éme Cycle, Université Aix-en Provence, Marseille, 178 p, 1987.

2. AIT BRAHIM, L., (1991) Tectonique cassante récente au nord du Maroc. Contribution à l'étude du risque sismotectonique, Thèse de Doctorat, Université Mohamed V, Rabat, 223 p, 1991.

3. AMRAOUI, F., (2005). Contribution à la connaissance des aquifères karstiques : cas de Lias de la plaine de Saïss et du Causse moyen atlasique tabulaire (Maroc), Thèse de Doctorat, Université Hassan II Aïn Chock, Casablanca, 227 p, 2005.

4. BOUMIR, K., (1987). Nouvelle interprétation du mode de mise en place des sables fauves dans le bassin du Saïss (Maroc). Thèse 3ème 
Cycle, Université Sidi Mohammed Ben Abdellah, Fès, Maroc, 169p, 1987.

5. ESSAHLAOUI, A., (2000). Contribution a la reconnaissance des formations aquifères dans le bassin de Meknès Fès, prospection géoélectrique étude hydrogéologique et intervenir des ressources en eau, Thèse de Doctorat, Université Mohamed V, Rabat, 258 p,2000.

6. FASSI, D., (1999). Les formations superficielles du Saïss de Fès et de Meknès. Du temps géologique à l'utilisation actuelle des sols, Notes et Mémoire de Service Géologique, 389, 524 p, 1999.

7. HARMOUZI, O., (2010). Reconnaissance détaillée de la partie nordest du Bassin de Saïss (MAROC): interprétation de sondages électriques verticaux par combinaison des méthodes statistique, géostatistique et d'inversion, thèse de doctorat, Université Moulay Ismail, Meknès, 304 p, 2010.

8. OURGHACHE, D., (1987). Etude géologique dans le Paléozoïque et le Trias de la bordure NW du causse moyen atlasique (S et SW de Fès, Maroc), thèse de 3éme cycle, Université de Paul Sabatier, Toulouse, $130 \mathrm{p}, 1987$.

9. TALTASSE, P.,(1953). Recherches géologiques et hydrogéologiques dans le bassin lacustre de Fès-Meknès. Notes et Mémoire de Service Géologique, 115, 300 p, 1953.

10. Leclerc, G., Bonneau, A., (1982). La lixiviation : source, composition et atténuation. Eau du Québec, 15, (1) ,37-45p, 1982.

11. Matejka, G., Rink, M., Mejebri, R., Bril, H., (1994). Pollution engendrée par un lixiviat de décharge d'ordure ménagère: Bilan hydrique et caractérisation. Environnemental technology, 15, 313322p, 1994.

12. Navarro, A., Bernard, D., Millot, N., (1998). Les problèmes des pollutions par les lixiviats de décharge. Technique, science et méthodes - L'eau, 3, 541-545p, 1998.

13. Chofqi, A., Younsi, A., Lhadi, E., Mania, J., Mudry, J., Veron, A., (2003). Pollution d'une nappe phréatique par les métaux lourds du lixiviat de décharge (El Jadida, Maroc). $2^{\text {ème Journées des géosciences }}$ de l'environnement, Université Ibn Tofail, Kenitra, Maroc, 2003.

14. TSM., (1990). Les lixiviations des décharges : le point des connaissances en 1990, TSM, 6/90, p 289-314, 1990.

15. Hakkou, R., (2001). La décharge publique de Marrakech: caractérisation des lixiviats, étude de leur impact sur les ressources en eau et essai de leur traitement. Thèse de doctorat d'état, université de CAPI AYYAD, Maroc, 150p, 2001. 
16. SITA., (2013). Etude d'impact sur l'environnement de la création et l'exploitation du centre d'élimination et de valorisation des déchets de la ville de Meknès, 150p, 2013.

17. ANRED., (1986). Etude du bilan hydrique des décharges industrielles, 120p, 1986.

18. CID/SEURECA., (2006). Etude d'actualisation du schéma directeur d'assainissement liquide de l'agglomération de la ville de Meknès, $197 \mathrm{p}, 2006$.

19. GTZ., (2010). Etude sur les possibilités d'une séparation des déchets en considérant les aspects techniques, économiques, écologiques de la nouvelle décharge de la ville de Meknès, Maroc, 94p, 2010.

20. Tahiri, A., Laziri, F., (2014). Etude des polluants contenus dans les lixiviats issus de la décharge publique de la ville de Meknès (Maroc), 170-186 (2014).

21. Site internet :

http://www.diastratasa.com/environnement/decharges.htm 